\title{
IMPACTOS AOS ATRIBUTOS FíSICOS DO SOLO EM TRILHAS OCASIONADOS PELO ECOTURISMO EM UBATUBA-SP
}

\author{
IMPACTS ON PHYSICAL ATTRIBUTES OF SOIL ON TRAILS ASSOCIATED WITH \\ ECOTOURISM IN UBATUBA, SP
}

\author{
Adriana Cristina Rosa Saraiva ${ }^{1}$
}

RESUMO: Em reservas florestais, a utilização de trilhas pelo trânsito de pessoas pode provocar destruição da camada de detritos vegetais sobre a superfície do solo, alterando seu estado estrutural e, dessa forma, causar a compactação do solo. O objetivo deste trabalho foi analisar os níveis de compactação em diferentes posições da Trilha dos Escravos, especificamente, centro e bordas, comparando-os com pontos existentes em condições ideais no interior da mata na Agência Paulista de Tecnologia de Agronegócios - APTA, em Ubatuba-SP. Foram amostrados 10 pontos, num percurso de 500 metros da trilha nas posições: centro, borda direita, borda esquerda e interior da mata. Em cada ponto amostral, foram coletadas amostras de solo para avaliação das propriedades físicas da umidade gravimétrica, densidade do solo, porosidade total e resistência à penetração. $O$ estudo demonstrou que existe uma diferença significativa entre as posições locadas, os níveis de compactação do centro da trilha, ocasionados pelo pisoteio, são superiores aos dos pontos locados nas bordas e no interior da mata. Os resultados também demonstram o efeito nocivo da ação do pisoteio no aumento da resistência à penetração do solo devido à utilização da trilha pela visitação pública, sendo necessárias, como medidas para a conservação da Trilha dos Escravos, um bom planejamento e educação ambiental aos visitantes.

Palavras-chave: compactação do solo; ecoturismo; impacto em trilhas.

ABSTRACT: In forest reserves, the use of trails by people can cause depletion of the plant debris on the soil surface, altering its structural state and thus cause soil compaction. The aim of this study was to analyze the level of compaction in different positions of the Slave Trail, specifically, the center and edges, comparing them to existing points in ideal conditions inside the forest in the Paulista Agency of Agribusiness Technology - APTA, in Ubatuba- SP. We sampled 10 points, 500 meters apart on the trail at the center, right edge, and left edge, as well as the forest interior. At each sample point, soil samples were collected for evaluation of physical properties including gravimetric moisture, density, porosity, and penetration resistance. The study demonstrated that there is a significant difference between the areas. The levels of compaction at the center of the trails, caused by trampling of people, are superior to those at the edges and inside the forest. The results also demonstrate the harmful effect of trampling on increasing the penetration resistance of soil due to the use of the trail for public visitation. Good planning and environmental education of the visitors are necessary to conserve the Slave Trail.

Keywords: soil compaction; ecotourism; impact on trails.

\footnotetext{
1 Pós-graduanda em Engenharia Ambiental pela UNINOVE e Mestre em Ciências Ambientais pela UNITAU. E-mail: dricacrisro@yahoo.com.br.
} 


\section{INTRODUÇÃO}

A principal atividade realizada em trilhas é o desenvolvimento de caminhadas de curtas a longas distâncias. As trilhas servem, ainda, como caminho aos atrativos ecoturísticos, além de serem utilizadas pelas populações residentes, dentro ou no entorno das Unidades de Conservação, a fim de atenderem suas necessidades de subsistência, tais como ligação entre sítios de agricultura e as residências, extrativismo, pesca etc. Essa diversidade de usos é fator relevante, seja para o visitante (ecoturista) ou para a população residente, já que a perpetuação do traçado em trilha é assegurada na prática das ações sobre ela (CASTRO, 2004).

As florestas tropicais possuem uma diversidade de hábitats que promove uma diversificação das comunidades vegetais e de recursos disponíveis para a fauna, contribuindo, portanto, para a excepcional riqueza de espécies, tanto de plantas como de animais (TUOMISTO et al., 1995), levando o homem à busca desse contato mais direto.

A ocorrência de impactos, nas trilhas, em áreas naturais, é consequência do uso, seja ele realizado com objetivos educacionais ou recreativos. Todos os visitantes, inclusive os mais conscientes, deixam pegadas (BARROS, 2003), ocasionando um conjunto de impactos, como expansão excessiva da largura, fuga da fauna nativa, ponto de concentração de lama, perda de matéria orgânica e vegetal. (ANDRADE, 2005)

As alterações dos atributos físicos do solo, em decorrência do pisoteio em trilhas utilizadas pelo ecoturismo, leva à compactação do solo e, consequentemente, ocorre incremento na densidade do solo, com diminuição da porosidade total, levando a um aumento na resistência à penetração, com redução na infiltração de água e aumento dos processos erosivos. Outra consequência inerente à ação erosiva é a redução da fertilidade do solo, devido às perdas de nutrientes.

Sendo assim, o objetivo geral deste trabalho foi de evidenciar a existência de alterações nos atributos físicos do solo da "Trilha dos Escravos", ocasionados pelo trânsito de pessoas, por meio do estudo das variações da densidade do solo, porcentagem da porosidade total $e$ resistência à penetração do solo nas camadas superficial e sub-superficial, em diferentes posições do centro e das bordas, direita e esquerda da Trilha dos Escravos, utilizadas pelos visitantes na APTA de Ubatuba-SP, comparando-as com as condições ideais do interior da mata.

\section{METODOLOGIA}

\subsection{Local de estudo}

A Agência Paulista de Tecnologia de Agronegócios - APTA, em Ubatuba-SP ou Instituto Agrônomo, como Estação Experimental de Ubatuba, localizado em uma área que proporciona aos turistas passeios ecológico-culturais, com destaque a "Trilha dos Escravos", que se inicia na sede do IAC - Instituto Agrônomo de Campinas, penetrando no interior da reserva com cobertura de Mata Atlântica, em um percurso de, aproximadamente, duas horas e meia de caminhada.

A área abrangida pela APTA-UPDU 
(Unidade de Desenvolvimento Tecnológico de Ubatuba) localiza-se no município de Ubatuba-SP, rodovia Oswaldo Cruz, 5061, $\mathrm{Km} \mathrm{88}$, com 427 ha, sendo $86 \%$ recoberto por Mata Atlântica, nas coordenadas $23^{\circ} 26^{\prime}$ $14^{\prime \prime}$ de latitude sul e $45^{\circ} 05^{\prime} 09^{\prime \prime}$ de longitude, a oeste de Greenwich. Encontra-se na interzona da planície litorânea (14\%) com as escarpas da Serra do Mar (86\%), num intervalo de altitude de 8 a 180 metros.

A vegetação é composta, basicamente, pela mata de encosta (baixa montana), em pequena área pela mata de planície com palmáceas (pupunha, jussara, açaí); arbórea de pequeno porte (cacau), arbórea de grande porte (seringueira) e arbustiva (mandioca). A mata de encosta e ciliar pluvial é constituída de árvores de grande porte com a presença de sub-bosque característico de mata primária.

Os solos existentes, na área da APTA de Ubatuba, são predominantemente do Grande Grupo CAMBISSOLO, Sub-Grupo CAMBISSOLO HÁPLICO (CX).

O clima da região, com base na classificação de Koppen, é do tipo Cwa, correspondente a um clima quente com invernos secos.

\subsection{Metodologia de Avaliação}

A avaliação do impacto em trilhas foi feita utilizando a metodologia proposta por Magro (1999) e Hammitt e Cole (1998), visando a identificar as alterações dos impactos. Foram amostrados 10 pontos, num percurso de 500 metros da trilha e nas posições: centro, borda direita, borda esquerda e interior da mata. Foram coletadas, nas parcelas, amostras de solo na profundidade $0-10 \mathrm{~cm}$ para avaliação da densidade do solo, umidade gravimétrica e porosidade total. A porosidade total do solo foi determinada por intermédio da porcentagem da porosidade total, utilizandose da equação de relação de densidades, na qual:

$$
\text { Pt\% }=\frac{(1-\text { Densidade do solo })}{\text { Densidade das partículas }} \times 100
$$

A avaliação da resistência do solo foi feita até a profundidade de $50 \mathrm{~cm}$, por meio de um penetrômetro de impacto. $A$ análise estatística dos dados foi realizada com a aplicação do teste $f$ e teste Tukey de comparação de médias, para $5 \%$ e $1 \%$ de probabilidade.

As parcelas foram escolhidas procurando-se manter a homogeneidade em relação à declividade, comprimento de rampa e forma de rampa.

\section{RESULTADOS E DISCUSSÃO}

A composição granulométrica do solo caracteriza-o como sendo da classe textural franco argilo arenoso, com base no diagrama de classes texturais adotado pela Sociedade Brasileira de Ciência do Solo (KIEHL, 1979). Como pode ser verificado, na Tabela 1, com os elevados teores da fração de areia seguida pela fração de argila. 
Tabela 1 - Variação da análise textural em diferentes posições da "Trilha dos Escravos" na APTA de Ubatuba-SP

\begin{tabular}{cccc}
\hline Posições & \%Areia Total & \%Silte & \%Argila \\
\hline Centro & 58,13 & 21,30 & 20,60 \\
Borda & 56,75 & 19,05 & 24,00 \\
Mata & 67,92 & 15,85 & 16,22 \\
Média & 60,93 & 18,73 & 20,27 \\
\hline
\end{tabular}

De acordo com a classificação textural do Embrapa (1999), os valores obtidos das frações granulométricas determinam a classe do solo como de textura média, tanto nos pontos locados nas posições centrais da trilha como nas bordas e no interior da mata, com teores da porcentagem de areia $>15 \%$ e de argila < 35\%. Essa classificação caracteriza o solo como de boa porosidade, quando em condições de cobertura vegetal de mata primária, significando que possui bom desenvolvimento radicular das plantas.

O resultado de um trabalho realizado por Castro (2004), na trilha da Cachoeira no Parque Estadual Turístico do Alto Ribeira, com a utilização do mesmo diagrama das classes texturais, apresentou resultados diferentes com o solo, e enquadrando-se na da classe de franco arenoso. Nesse tipo de solo, houve um aumento na porcentagem de silte e uma diminuição na de argila. Como era uma área submetida ao pisoteio, houve uma diminuição na quantidade de poros entre as partículas (inferior a 40\%). De acordo com Magro (1999), essa redução apresenta efeitos diretos no sucesso de germinação e no vigor das plantas.

Analisando as variações da resistência à penetração do solo, observou-se que os valores mais elevados foram na posição do centro da trilha, chegando a 4,81 $\mathrm{MPa}$, na camada abaixo de $30 \mathrm{~cm}$ (Tabela 2), o que se enquadra, segundo Arshad et al. (1996), em uma classe muito alta, com valores de 4,0 a 8,0 MPa. Na posição de borda esquerda, com valores de 3,55 MPa e para a borda direita, de 3,26 MPa (Tabela 2), esses resultados representam, para Arshad et al. (1996), uma classe alta com valores entre 2,0 a 4,0 MPa, considerados restritivos para o desenvolvimento radicular. 
Tabela 2 - Variação da resistência à penetração do solo em diferentes profundidades em (MPa) das posições da "Trilha dos Escravos" na APTA de Ubatuba-SP

\begin{tabular}{ccccc}
\hline $\begin{array}{c}\text { Profundidade } \\
(\mathbf{c m})\end{array}$ & Centro & $\begin{array}{c}\text { Posições na trilha } \\
\text { esquerda }\end{array}$ & Borda direita & $\begin{array}{c}\text { Interior da } \\
\text { mata }\end{array}$ \\
\hline $0-5$ & $1,32 \mathrm{~A}$ & $1,16 \mathrm{~A}$ & $1,35 \mathrm{~A}$ & $0,66 \mathrm{~B}$ \\
$5-10$ & $2,10 \mathrm{~A}$ & $1,77 \mathrm{~A}$ & $1,98 \mathrm{~A}$ & $1,11 \mathrm{~B}$ \\
$10-15$ & $2,94 \mathrm{~A}$ & $2,31 \mathrm{AB}$ & $2,30 \mathrm{AB}$ & $1,40 \mathrm{~B}$ \\
$15-20$ & $3,64 \mathrm{~A}$ & $2,52 \mathrm{AB}$ & $2,43 \mathrm{AB}$ & $1,61 \mathrm{~B}$ \\
$20-25$ & $3,79 \mathrm{~A}$ & $2,71 \mathrm{AB}$ & $2,64 \mathrm{AB}$ & $1,68 \mathrm{~B}$ \\
$25-30$ & $4,02 \mathrm{~A}$ & $2,90 \mathrm{AB}$ & $2,75 \mathrm{AB}$ & $1,77 \mathrm{~B}$ \\
$30-35$ & $4,19 \mathrm{~A}$ & $3,09 \mathrm{AB}$ & $2,93 \mathrm{AB}$ & $1,88 \mathrm{~B}$ \\
$35-40$ & $4,44 \mathrm{~A}$ & $3,23 \mathrm{AB}$ & $3,08 \mathrm{AB}$ & $1,98 \mathrm{~B}$ \\
$40-45$ & $4,70 \mathrm{~A}$ & $3,43 \mathrm{AB}$ & $3,22 \mathrm{AB}$ & $2,06 \mathrm{~B}$ \\
$45-50$ & $4,84 \mathrm{~A}$ & $3,57 \mathrm{AB}$ & $3,31 \mathrm{BC}$ & $2,10 \mathrm{C}$ \\
$50-55$ & $4,81 \mathrm{~A}$ & $3,55 \mathrm{AB}$ & $3,26 \mathrm{BC}$ & $2,12 \mathrm{C}$ \\
\hline
\end{tabular}

Médias seguidas por letras distintas diferem entre si ao nível de $1 \%$

Os dados demonstram que, nas posições central e bordas da trilha, os valores da resistência à penetração do solo, encontrados abaixo da camada de $30 \mathrm{~cm}$, são consequências do pisoteio sucessivo (Fig. 1), o que foi o principal responsável pela destruição da estrutura do solo original e, consequente, aumento de sua susceptibilidade à erosão (MAGRO, 1999).
Já, na posição de interior da mata, os valores estiveram dentro da interpretação de classe moderada, segundo Arshad, Lowery e Grossman (1996), entre 1,0 a 2,0 MPa (Fig. 1), valores que, também, podem ser considerados prejudiciais para 0 desenvolvimento radicular, mas, comparado com posições central e bordas, a resistência à penetração do solo do interior da mata apresenta boas condições de conservação. 


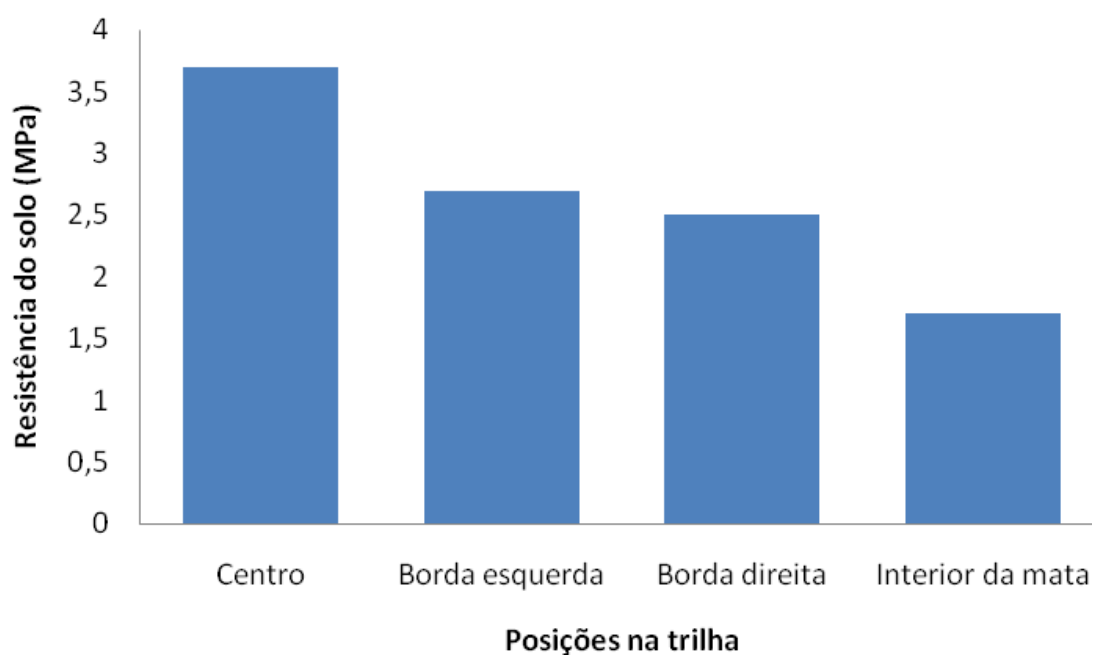

Fig. 1 - Relação da resistência à penetração do solo em (MPa) em diferentes posições da "Trilha dos Escravos" na APTA de Ubatuba-SP.

Takahashi (1998) e Takahashi, Milano e Tormena (2005) observaram que a resistência à penetração, a uma profundidade entre 5 e $10 \mathrm{~cm}$, na área da Reserva Natural de Salto Morato, foi a variável que estabeleceu a melhor discriminação entre a trilha e a área sem uso. Também, no Parque Estadual Pico do Marumbi - PR, a autora analisou duas áreas: na primeira clareira utilizada pelo público, a resistência à penetração foi média na superfície; na segunda, área de testemunha, sem o uso público, a resistência foi menor, havendo diferença significativa nos dois casos.

Takahashi (1998) argumenta que esses resultados ratificam a afirmação de outros autores de que a resistência à penetração é um indicador bastante sensível e valioso para 0 monitoramento das condições do solo.

A compactação do solo pode ser avaliada pela sua densidade, que pode atingir níveis críticos na camada superficial, em áreas impactadas. A densidade do solo reflete a agregação das partículas e os agregados do solo. A sua diminuição se reflete na maior capacidade de agregação com aumento na porosidade que favorece a retenção de água, o crescimento das raízes, as trocas gasosas e a atividade microbiana (ALVARENGA; SOUZA, 1998).

Observa-se que os valores encontrados para a densidade superficial do solo foram menores na posição de interior da mata, com 0,97 g/ $\mathrm{cm}^{3}$, (Tabela 3) e, por possuir menor valor, tem maior quantidade de poros. Para valores de densidade inferiores a $1,00 \mathrm{~g} / \mathrm{cm}^{3}$, são considerados de uma classe muito alta e representam a condição ideal para o desenvolvimento radicular; já, nas posições de borda esquerda, com $1,16 \mathrm{~g} / \mathrm{cm}^{3}$; e borda direita, com 1,23 $\mathrm{g} / \mathrm{cm}^{3}$, são considerados, por Greenland (1981), como de classe média para 0 desenvolvimento radicular com valores entre 1,14 e $1,27 \mathrm{~g} / \mathrm{cm}^{3}$. 
Tabela 3 - Variação da porosidade total, densidade do solo e umidade nas diferentes posições da "Trilha dos Escravos" na APTA de Ubatuba-SP

\begin{tabular}{cccc}
\hline Posição na trilha & $\begin{array}{c}\text { Porosidade total } \\
\text { (\%) }\end{array}$ & $\begin{array}{c}\text { Densidade do solo } \\
\mathbf{g} / \mathbf{c m}^{\mathbf{3}}\end{array}$ & $\begin{array}{c}\text { Umidade } \\
\text { (\%) }\end{array}$ \\
\hline Mata & $64,50 \mathrm{~A}$ & $0,97 \mathrm{C}$ & $48,39 \mathrm{~A}$ \\
Borda esquerda & $56,84 \mathrm{~B}$ & $1,16 \mathrm{~B}$ & $44,04 \mathrm{~A}$ \\
Borda direita & $54,30 \mathrm{~B}$ & $1,23 \mathrm{~B}$ & $43,69 \mathrm{~A}$ \\
Centro & $43,83 \mathrm{C}$ & $1,46 \mathrm{~A}$ & $45,07 \mathrm{~A}$ \\
& $\mathrm{P}>\mathrm{F}=0,001$ & $\mathrm{P}>\mathrm{F}=0,001$ & $\mathrm{P}>\mathrm{F}=0,85$
\end{tabular}

Médias seguidas por letras distintas diferem entre si ao nível de 1\%

Já, em relação à posição central da trilha, observou-se um aumento na densidade do solo, com valor médio de 1,46 $\mathrm{g} / \mathrm{cm}^{3}$ (Tabela 3), que, segundo Greenland (1981), encontra-se no intervalo de 1,27 e $1,47 \mathrm{~g} / \mathrm{cm}^{3}$, considerado como de classe baixa para o desenvolvimento radicular, pois resulta em uma diminuição dos poros. Esses resultados estão de acordo com o que Vallim et al. (2007) observaram em sua pesquisa na trilha de intensa visitação, no Maciço Gericinó-Mendanha - RJ. Os autores encontraram valores, para a densidade do solo, maiores de $1,4 \mathrm{mg} \mathrm{m}^{-3}$, enquanto que, na trilha de menor visitação, foi $0,80 \mathrm{mg} \mathrm{m}^{-3}$. Vashchenko (2006), nos Picos Camapuã e Tucum - Campina Grande do Sul - PR, também estudando um solo do tipo CAMBISSOLO HÁPLICO, encontrou valores de densidade do solo de $0,76 \mathrm{mg} \mathrm{m}^{-3}$ na trilha e de $0,54 \mathrm{mg} \mathrm{m}^{-3}$ fora da trilha. Esses valores são considerados de classe muito alta, representando boas condições para o desenvolvimento das plantas. Esses estudos foram realizados em condições de solo e cobertura vegetal semelhantes às encontradas na APTA de Ubatuba, objeto da presente pesquisa.

Os dados da porosidade que estão na média de $44 \%$ na posição central da trilha, $57 \%$ nas posições de bordas e $64,5 \%$ no interior da mata (Tabela 3), estão de acordo com o intervalo adequado de variação para os solos de textura média, citados por Rosolem et al. (1994) que é de $45 \%$ a $65 \%$. Com o trabalho de Vashchenko (2006), encontrou-se em um solo do tipo CAMBISSOLO HÁPLICO uma porosidade total de $65,48 \%$ na trilha, e uma porosidade total de $75,5 \%$ fora da trilha, concluindo-se que, de maneira geral, a porosidade total fora da trilha foi maior que a porosidade total na trilha, podendo-se afirmar, com base nesses dados, que quanto menor a densidade do solo maior sua porosidade total.

Em relação à umidade, não houve diferenças significativas entre os pontos locados. Na literatura, também não existe um intervalo de variação recomendado, pois depende das condições climáticas da região, assim também como foi constatado por 
Magro (1999), em sua pesquisa no Parque do Itatiaia. A autora avaliou o conteúdo de umidade do solo, por meio de avaliação visual no local, no mesmo período de estudo de dois anos, em que foi realizada a coleta dos dados de resistência e densidade do solo.

O uso recreativo de trilhas, pelo pisoteio, age sobre a estrutura do solo, diminuindo sua porosidade e aumentando sua densidade, levando a um aumento da resistência à penetração, o que é prejudicial ao crescimento radicular e perda da cobertura vegetal, ocasionando processos erosivos, como foi apresentado na Tabela 1, e conforme Takahashi (1998), Vashchenko (2006) e Vallim, Xavier e Gama (2007).

\section{CONCLUSÃO}

$\mathrm{Na}$ posição de interior da mata, todas as amostras analisadas não apresentaram problemas relacionados à compactação. Os valores de densidade do solo, porosidade total e resistência à penetração do solo estão dentro do que se considera condições ideais para o desenvolvimento radicular das plantas. $\mathrm{Na}$ posição central e bordas da trilha, a camada abaixo de $30 \mathrm{~cm}$ de profundidade, evidenciada pelo seu elevado valor da resistência à penetração do solo, apresentou restrições ao desenvolvimento radicular, levando, também, a alterações no valor de densidade do solo que promoveu redução na quantidade de poros responsáveis pela circulação de ar no solo. A utilização de trilhas, em áreas naturais, provoca, ao longo do tempo, uma compactação na camada superficial do solo, dificultando a infiltração da água e favorecendo a ocorrência de erosão superficial que promove perdas de nutrientes. Um bom plano de manejo e educação ambiental aos visitantes seria uma das medidas a ser tomada para a conservação da Trilha dos Escravos, que representa um valor cultural, histórico e ambiental tão necessário à humanidade, nos dias de hoje.

Recomenda-se a realização de estudos de capacidade de carga antes da locação e aberturas de trilhas em áreas de preservação ambiental.

\section{REFERÊNCIAS}

ALVARENGA, M. I. N.; SOUZA, J. A. Atributos do solo e o impacto ambiental. Lavras: Fundação de Apoio ao Ensino, pesquisa e extensão. Universidade Federal de Lavras, 1998. 205p.

ANDRADE, A. J. Manejo de trilhas para o ecoturismo. In NEIMAN, Z. Ecoturismo no Brasil. São Paulo: Manole, 2005. p. 131151.

ARSHAD, M. A.; LOWERY, B.; GROSSMAN, $B$. Physical tests for monitoring soil quality. In: DORAN, J. W.; JONES, A. J. (Ed.). Methods for assessing soil quality. Madison : Soil Science Society of America, 1996. p. 123-141. (SSSA Special Publication, 49).

BARROS, M. I. Caracterização da visitação, dos visitantes e avaliação dos impactos ecológicos e recreativos do planalto do parque nacional do Itatiaia. 2003. $121 \mathrm{p}$. Dissertação (Mestrado) - Escola Superior de Agricultura Luiz de Queiroz, Universidade de São Paulo, Piracicada, 2003.

CASTRO, C. E. O caminho entre a percepção, o impacto no solo $e$ as 
metodologia de manejo. O estudo de trilhas do Parque Estadual Turístico do Alto RibeiraSP. 2004. 153p. Dissertação (Mestrado). Universidade Estadual de Londrina, Londrina, 2004.

EMBRAPA Centro Nacional de Pesquisa de Solos. Sistema Brasileiro de Classificação de Solos. Rio de Janeiro: Embrapa Solos, 1999. 412p.

GREENLAND, D. J. Soil management and soil degradation. Journal of Soil Science, London, v. 31, n. 3, p. 301-322, 1981.

HAMMITT, W. E.; COLE, D. N. Wildland recreation: ecology and management. New York: John Wiley, 1998. 361p.

KIEHL, J. E. Manual de Edafologia. São Paulo: Editora Agronômica Ceres, 1979.

MAGRO, T. C. Impactos do uso público em uma trilha no planalto do Parque Nacional do Itatiaia. 1999. 135p. Tese (Doutorado) Escola de Engenharia de São Carlos, Universidade de São Paulo, São Carlos, 1999.

ROSOlEM, C. A.; ALMEIDA, A. C. S.; SACRAMENTO, L. V. S. Sistema Radicular e nutrição da soja em função da compactação do solo. Bragantia, Campinas. v. 53, n. 2, p. 259-266, 1994.
TAKAHASHI, L. Y. Caracterização dos visitantes, suas preferências e percepções e avaliação dos impactos da visitação pública em duas unidades de conservação do estado Paraná. 1998. Tese (Doutorado em Engenharia Florestal). Setor de Ciências Agrárias, Universidade Federal do Paraná, Curitiba, 1998.

TAKAHASHI, L. Y.; MILANO, M. S.; TORMENA, C. A. Indicadores de impacto para monitorar o uso público no Parque Estadual Pico do Marumbi-PR, Revista Árvore, Viçosa, v. 29, n. 1, p. 159-167. 2005.

TUOMISTO, H. et al. Dissecting Amazonian biodiversity. Science, v. 269, p. 63-66. 1995.

VALLIM, C. M. C.; XAVIER, T. F.; GAMA, S. V. G. 2007. Trilhas: solo, vegetação e probelmas decorrentes do uso - o caso do maciço Gericinó-Mendanha (RJ). Rio de Janeiro: Universidade do Estado do Rio de Janeiro. Disponível em: $<$ http://www.igeo.uerj.br/VICBG-

2004/Eixo2/E2_161.htm>. Acesso em: jul. 2007.

VASHCHENKO, Y. Caracterização da trilha e o impacto do montanhismo nos Picos Camapuã e Tucum: Campina Grande do Sul - PR, 2006. 106p. Dissertação (Mestrado)Ciências Agrárias, Universidade Federal do Paraná, Curitiba, 2006. 